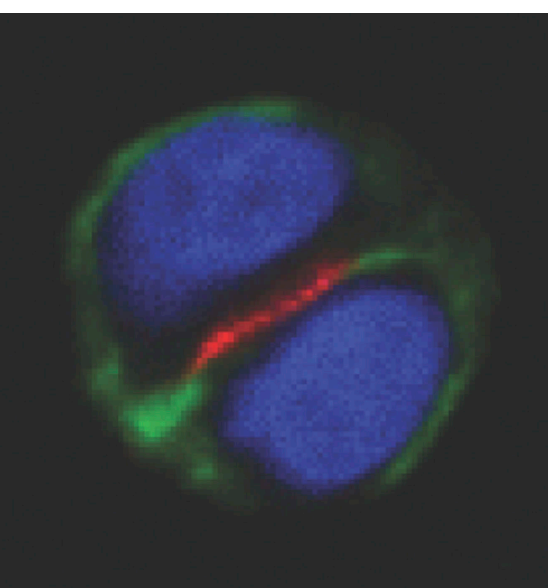

Epithelial cells are already polarized by the first division, as indicated by the presence of the apical marker ZO-1 (red) on the cells' inner surfaces.
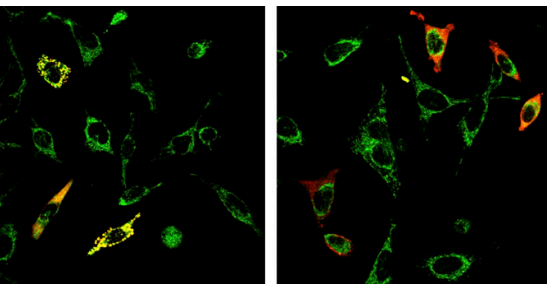

Caspase-8 (red) normally homes in on mitochondria (green) but diffuses widely when mature cardiolipin is absent (right).

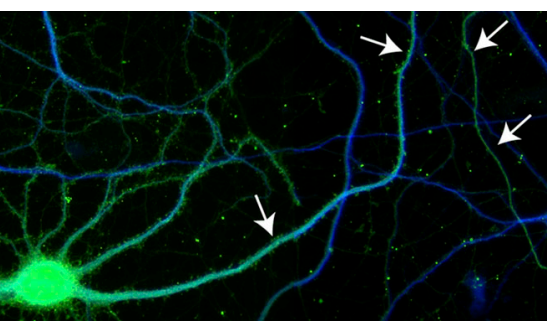

After removal of ankyring, this axon (arrows) grew a stubble of dendritic spines.

\section{Cdc42 turns the spindle}

During development, epithelial cells need a little help figuring out which way is up. Jaffe et al. show how a polarizing protein ensures that the cells face the right direction.

A newborn epithelial cell in, say, the intestinal lining will polarize so that its apical surface is oriented toward the gut lumen. The protein $\mathrm{Cdc} 42$ helps set up polarity in a range of organisms and cell types, and previous studies suggested that it does the same in epithelial cells. The mechanism remained obscure, however.

To determine how Cdc42 differentiates top from bottom, Jaffe et al. grew human intestinal cells in a three-dimensional culture. The cells divided repeatedly to form a sphere, with their apical surfaces directed toward a central lumen.

When the researchers used RNAi to slash Cdc42 levels, they expected the cells to become symmetric. Instead, the cells polarized, but they clustered around multiple lumens. That result indicates that rather than instigating polarization, $\mathrm{Cdc} 42$ determines where it occurs.

Cdc42 orients cells by helping position the mitotic spindle so that it is perpendicular to the middle of the sphere. Thus, division splits each cell radially, and the apical surface of each daughter remains in the center of the cluster. If $\mathrm{Cdc} 42$ is missing, the spindle orients randomly, and the apical surface of a particular daughter cell can end up outside the center-hence, the extra lumens.

The researchers also found that the apical surface forms at the last point of contact between dividing cells. They showed that the midbody, the tether between daughter cells, is located asymmetrically so that all apical surfaces form at the center of the cluster. Determining whether Cdc42 controls midbody position will require further research.

Jaffe, A.B., et al. 2008. J. Cell Biol. doi:10.1083/icb.200807121.

\section{A get-together for caspases}

Before they can get down to killing a cell, apoptosis-promoting caspase-8 enzymes must socialize. Gonzalvez et al. reveal that a mitochondrial lipid provides a gathering place where the enzymes can mingle.

After receiving an external death signal, cells can commit suicide in several ways. In one mechanism, inactive copies of caspase- 8 gather at the death-inducing signaling complex (DISC) on the cell membrane. There, the molecules combine and cut one another, becoming active enzymes.

But in an alternative mechanism, Gonzalvez et al. showed that caspase- 8 migrates to the mitochondria instead of combining at the DISC. While probing the function of cardiolipin, a mitochondrial membrane lipid, Gonzalvez et al. discovered how caspase- 8 molecules meet up at this organelle.

The researchers tested cells from patients with Barth syndrome, a rare genetic disorder in which the functional form of cardiolipin is scarce. The cells survived treatment with apoptosis-triggering molecules such as TNF $\alpha$. Without mature cardiolipin, caspase- 8 doesn't embed in the mitochondrial membrane and doesn't get activated, the scientists found.

Cardiolipin seems to serve as the mitochondrial equivalent of the DISC, a platform where caspase- 8 molecules can collect and undergo the modifications necessary for activation. Still unclear is what signal directs caspase- 8 to the mitochondria and whether other caspases also travel to the organelles to be switched on.

Gonzalvez, F., et al. 2008. J. Cell Biol. doi:10.1083/jcb.200803129.

\section{Axons lose their identity}

Inside every axon is a dendrite waiting to get out. Hedstrom et al. converted mature axons into dendrites by banishing a protein crucial for neuron development. The results suggest that this transformation could occur after nerve cell damage.

At the junction between the axon and the cell body is the axon initial segment (AIS), which sparks action potentials. Researchers also suspect that the AIS enables an axon to maintain its identity. Although scientists have teased out some of the molecular events that determine whether a neuron outgrowth will become an axon or a dendrite, they know little about what keeps these structures distinct.

In cultured neurons, Hedstrom et al. used RNAi to cut production of ankyrinG, a protein that helps spur formation of the AIS. Loss of ankyrinG caused the AIS to disappear. For example, sodium channels 
that normally crowd the AIS's plasma membrane dispersed. When ankyrinG was absent the erstwhile axons began to resemble dendrites, sprouting spines and developing excitatory synapses. Characteristic molecules from the cell body and dendrites, such as the microtubule polymerizing protein MAP2, also infiltrated the axons.

The AIS appears to serve as a filter that screens out dendritic proteins, Hedstrom et al. conclude. How the structure bars some proteins from the axon is still uncertain. Injuries and diseases can transform dendrites into axons. The findings raise the possibility that these insults could cause the reverse transformation by changing the amount of ankyrinG and altering the AIS.

Hedstrom, K.L., et al. 2008. J. Cell Biol. doi:10.1083/jcb.200806112.

\section{Uniting to save cancer cells}

A colon cancer cell isn't a lost cause. Vitamin D can tame the rogue cell by adjusting everything from its gene expression to its cytoskeleton. Ordóñez-Morán et al. show that one pathway governs the vitamin's diverse effects. The results help clarify the actions of a molecule that is undergoing clinical trials as a cancer therapy.

Vitamin D stymies colon cancer cells in two ways. It switches on genes such as the one that encodes E-cadherin, a component of the adherens junctions that anchor cells in epithelial layers. The vitamin also induces effects on the cytoskeleton that are required for gene regulation and short-circuiting the Wnt/ $\beta$-catenin pathway, which is overactive in most colon tumors. The net result is to curb division and prod colon cancer cells to differentiate into epithelial cells that settle down instead of spreading.

To delve into the mechanism, the team dosed colon cancer cells with calcitriol, the metabolically active version of vitamin D. Calcitriol triggered a surge of calcium into the cells and the subsequent switching on of RhoA-RhoGTPases, which have been implicated in the cytoskeletal changes induced by vitamin D. The activated RhoA in turn switched on one of its targets, the rho-associated coiled kinase (ROCK), which then roused two other kinases. Each step in this nongenomic pathway was necessary to spur the genomic responses, the researchers showed. The team also nailed down the contribution of the vitamin D receptor (VDR). The receptor was crucial at the beginning of the pathway, where it permitted the calcium influx, and at the end, where it activated and repressed genes.

The study is the first to show that vitamin D's genomic and nongenomic effects integrate to regulate cell physiology. One question the researchers now want to pursue is whether VDR from different locations - the nucleus, the cytosol, and possibly the cell membrane-has different functions in the pathway.

Ordóñez-Morán, P., et al. 2008. J. Cell Biol. doi:10.1083/jcb.200803020.

\section{NA healer}

Aucott et al. report the first in vivo experiments on the heterochromatin protein 1 (HP1) family, which sidles up to silent DNA. The results add to the evidence that the different versions of the proteins help cells fix broken DNA.

The function of HP1 proteins has puzzled researchers. The proteins, which come in three forms in mammals, cozy up to heterochromatin - the tightly wound sections of DNA where genes are usually inactive. Early studies indicated that the proteins' job was to turn genes off. But recent work suggested that the proteins are essential for repairing damaged DNA. These results came from in vitro studies, however, and the proteins' powers in vivo remained uncertain.

Aucott et al.created the first mouse strain missing one of the HP1 versions, HP1 13 . The animals die shortly after birth because their lungs don't inflate. The rodents show brain defects as well. Large numbers of neurons die, for example, and the neural stem cells in the cortex divide sluggishly. Both effects could arise from unfixed DNA. When the researchers grew brain cells from HP1 $\beta$ lacking mice in culture, they saw clear indications of genomic instability that can result from faulty DNA repair, including unpaired sister chromatids that separated prematurely and even extra sets of chromosomes. The HP1 proteins latch onto the methylated version of the H3 histone, but how this interaction promotes repair is an unanswered question.

Aucott, R., et al. 2008. J. Cell Biol. doi:10.1083/jcb.200804041.
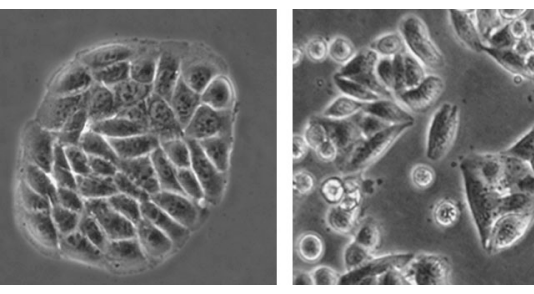

Vitamin D spurs cancer cells to take on the spread-out epithelial phenotype (left), but they are more rounded if RhoA is disrupted (right).

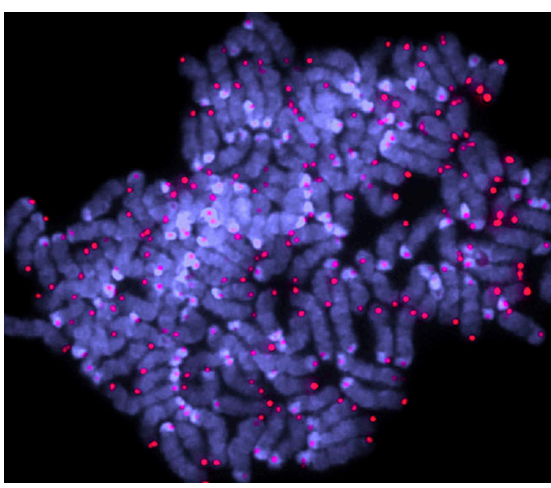

Extra chromosomes crowd a polyploid cell from a mouse lacking HP1ß. 